\title{
Raf kinase inhibitor protein is downregulated in hepatocellular carcinoma
}

\author{
MARION M. SCHUIERER ${ }^{1}$, FRAUKE BATAILLE ${ }^{1}$, THOMAS S. WEISS $^{2}$, \\ CLAUS HELLERBRAND ${ }^{3}$ and ANJA K. BOSSERHOFF ${ }^{1}$ \\ ${ }^{1}$ Institute of Pathology, ${ }^{2}$ Center for Liver Cell Research, ${ }^{3}$ Department of Internal Medicine I, \\ University of Regensburg, Franz-Josef-Strauss-Allee 11, 93053 Regensburg, Germany
}

Received January 13, 2006; Accepted March 28, 2006

\begin{abstract}
The Ras/Raf/MEK/ERK signalling cascade is frequently deregulated in tumourigenic diseases and known to be involved in proliferation and transformation of cells. Also in hepatocellular carcinoma (HCC) increased ERK levels are observed and known to correlate with tumour progression, but the underlying molecular mechanism are unknown. We analyzed expression of Raf-1 kinase inhibitory protein (RKIP) in HCC. Expression of RKIP mRNA and protein was downregulated in HCC cell lines and tissue as compared to primary human hepatocytes $(\mathrm{PHH})$ or non-tumorous liver tissue, respectively. Transfection of an HCC cell line with an RKIP expression construct blocked the Raf kinase pathway resulting in decreased activity of ERK1/2 and AP-1. In contrast, downregulation of RKIP by transfection with an antisense RKIP construct led to increased ERK1/2 and AP-1 activity. Since HCC develop in the majority of cases in cirrhotic liver tissue and cirrhosis is the main risk factor for HCC development, we analyzed RKIP expression also in non-cancerous cirrhotic liver tissues by immunohistochemistry. In contrast to normal liver tissue, where the staining was equally distributed within the cytoplasm, hepatocytes in cirrhotic liver revealed an intense RKIP staining of the membrane. It can be speculated that this changed RKIP expression pattern parallels impaired protein function in $\mathrm{PHH}$ in cirrhotic livers that may predispose $\mathrm{PHH}$ to malignant transformation. In addition, our study demonstrates functional relevance of downregulation of RKIP in HCC that may play an important role in HCC development and progression.
\end{abstract}

\section{Introduction}

Hepatocellular carcinoma (HCC) is one of the most common human malignancies and accounts for $>90 \%$ of all primary

Correspondence to: Dr Anja Bosserhoff, Institute of Pathology, University of Regensburg, Franz-Josef-Strauss-Allee 11, 93053 Regensburg, Germany

E-mail: anja.bosserhoff@klinik.uni-regensburg.de

Key words: hepatocellular carcinoma, Raf signalling, Raf kinase inhibitor protein, MAPK pathway liver cancers. In the majority of cases HCC develops in cirrhotic liver. Although associated etiological factors are recognized, interactions between individual factors and molecular mechanisms by which they lead to cancer remain largely unclear (1-4).

The Ras/Raf-1/mitogen-activated protein/extracellular signal-regulated kinase kinase (MEK)/extracellular signal regulated kinase (ERK) signalling pathway regulates multiple biological processes including mitogenesis and differentiation $(5,6)$. ERK is phosphorylated by MEK, which in turn is phosphorylated and activated by Raf, that itself gets phosphorylated upon activation of the small G-protein Ras. Phosphorylated ERK translocates into the nucleus and regulates gene expression via activation of various transcription factors $(7,8)$. The importance of this signalling cascade and its correct regulation implicates that deregulation may lead to malignant transformation of cells and induction of tumour development. Mutations in Ras, Raf, MEK, and ERK were identified in a variety of tumour entities and it has been shown that ERK is overexpressed and activated in a high number of malignant tumours including HCC. Furthermore, Ito and coworkers have shown that activation of ERK1/2 can be correlated to progression of HCC (9-12). The molecular mechanisms for constitutive activation of ERK in HCC are unknown so far; therefore, we analysed whether loss of the endogenous inhibitory protein RKIP contributes to constitutive ERK activity and malignant transformation of hepatocytes.

Raf kinase inhibitor protein (RKIP) is a cellular inhibitor of the MAP kinase cascade as it dissipates the Raf-1/MEK interaction, thereby preventing activation of MEK by Raf-1 and downstream signal transduction $(13,14)$. RKIP is a member of the phosphatidylethanolamine binding protein (PEBP) family, a ubiquitously expressed and evolutionarily conserved group of proteins (15). RKIP expression has been shown to be downregulated in metastatic prostate cancers and the loss of RKIP levels was suggested to promote the metastatic potential of prostate cancer cells (16). Furthermore, in a recent study we were able to show a decrease of RKIP expression in malignant melanoma and the absence of RKIP expression in melanoma metastases (17).

The aim of this study was to analyse RKIP expression in HCC and to investigate whether impaired RKIP may contribute to enhanced activity of the Ras/Raf/MEK/ERK signalling cascade in this cancer entity. 


\section{Materials and methods}

\section{Patients and patient material}

Human liver tissue. HCC tissue and non-tumorous liver tissue of the same patient were obtained from 15 HCC patients undergoing partial hepatectomy. Tissue samples for RT-PCR studies were immediately snap-frozen and stored at $-80^{\circ} \mathrm{C}$. For (immuno-) histological analysis, the liver tissue was formalinfixed.

Primary human hepatocytes. Tissue samples from human liver resections were obtained from patients undergoing partial hepatectomy for metastatic liver tumors of colorectal cancer. Only liver tissues judged microscopically as non-cancerous by an anatomical pathologist were used for cell preparation. Further exclusion criteria were: known liver disease or histological evidence for liver fibrosis or inflammation in surrounding non-tumorous liver tissue. Hepatocytes were isolated using a modified two-step EGTA/collagenase perfusion procedure as described previously (10). Informed consent was obtained from all patients and the study was approved by the local ethics committee.

HCC cell lines and tissue culture. The following HCC cell lines were used: HepG2 (ATCC HB-8065), PLC (ATCC CRL-8024), and Hep3B (ATCC HB-8064). Cells were grown at $37^{\circ} \mathrm{C} / 5 \% \mathrm{CO}_{2}$ in Dulbecco's modified Eagle medium (DMEM; PAN Biotech, Aidenbach, Germany) supplemented with penicillin $(100 \mathrm{U} / \mathrm{ml})$, streptomycin $(10 \mathrm{mg} / \mathrm{ml})$ (both Sigma, Deisenhofen, Germany), and $10 \%$ fetal calf serum (PAN Biotech), and split 1:4 at confluence. Cells were detached by incubation with $0.05 \%$ trypsin, $0.04 \%$ EDTA (Sigma) in PBS for $5 \mathrm{~min}$ at $37^{\circ} \mathrm{C}$. For demethylation assays the cells were treated for 24 or $48 \mathrm{~h}$ with 5-azacytidine (Sigma) at a final concentration of $10 \mu \mathrm{M}$ (17).

Transient transfection of HepG2 cells. HepG2 cells were transiently transfected with sense RKIP or with antisense RKIP expression plasmid (RKIP full length coding sequence cloned into pcDNA3.1 (Invitrogen, NV Leek, The Netherlands) in sense or antisense direction). Controls received pcDNA3.1 plasmid DNA. Transfections were performed using the Lipofectamin Plus method (Invitrogen, Karlsruhe, Germany). RKIP expression levels in these cells were determined by RT-PCR and Western blot analysis.

Luciferase reporter gene assays. For transfections, $2 \times 10^{5}$ cells per well were seeded into six-well plates and transiently transfected with $0.5 \mu \mathrm{g}$ of pAP-1 luc plasmid (Stratagene, La Jolla, USA) using the Lipofectamine Plus method (Invitrogen) according to the manufacturer's instructions. Twenty-four hours after transfection cells were lysed and the luciferase activity in the lysate was measured. To normalise transfection efficiency, $0.2 \mu \mathrm{g}$ of pRL-TK plasmid (Promega, Mannheim, Germany) was cotransfected and renilla luciferase activity was measured using a luminometric assay (Promega). All transfection experiments were repeated at least 3 times.

RNA isolation and reverse transcription. For RT-PCR total cellular RNA was isolated from cultured cells using the RNeasy kit (Qiagen). Integrity of RNA was controlled on a
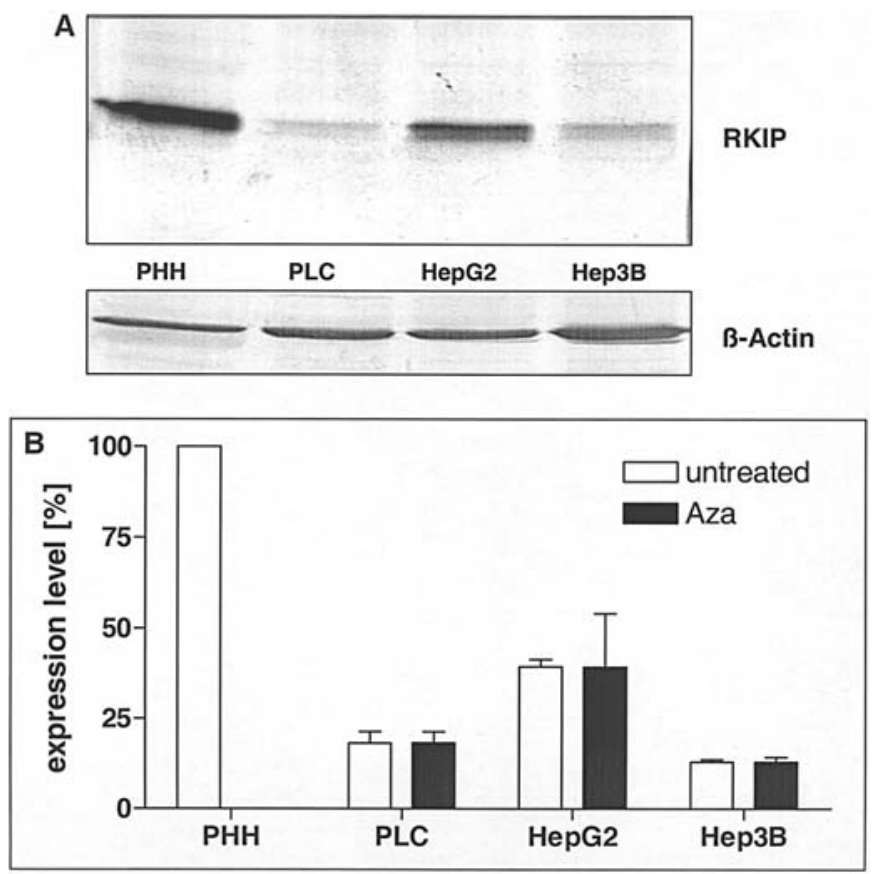

Figure 1. RKIP expression in HCC cell lines compared to primary human hepatocytes. (A) In 3 analysed HCC cell lines (PLC, Hep3B, HepG2) the expression of RKIP protein was reduced in comparison to primary human hepatocytes $(\mathrm{PHH})$. The Western blot was re-probed with an anti- $\beta$-actin antibody as loading control. (B) The amount of RKIP mRNA expression was quantified by real-time PCR. All 3 HCC cell lines PLC, HepG2, and Hep3B showed a strong reduction of RKIP expression compared to PHH (white bars). Treatment of the cell lines with the demethylating agent 5-azacytidine failed to significantly induce RKIP mRNA expression (black bars).

$1 \%$ agarose/formaldehyde gel. Subsequently cDNAs were generated by reverse transcriptase reactions. The reverse transcription (RT) reaction was performed in $20 \mu 1$ reaction volume containing $2 \mu \mathrm{g}$ of total cellular RNA, $4 \mu 15 \mathrm{X}$ first strand buffer (Invitrogen), $2 \mu 10.1 \mathrm{M}$ DTT, $1 \mu \mathrm{l} \mathrm{dN}_{6}$ primer $(10 \mathrm{mM}), 1 \mu \mathrm{ldNTPs}(10 \mathrm{mM})$, and DEPC water. The reaction mix was incubated for $10 \mathrm{~min}$ at $70^{\circ} \mathrm{C}$. Then $1 \mu \mathrm{l}$ of Superscript II RT (Invitrogen) was added and RNAs were transcribed for $1 \mathrm{~h}$ at $37^{\circ} \mathrm{C}$. RT was inactivated at $70^{\circ} \mathrm{C}$ for $10 \mathrm{~min}$ and RNA was degraded by digestion with $1 \mu 1 \mathrm{RNase}$ A (10 mg/ $\mathrm{ml}$ ) at $37^{\circ} \mathrm{C}$ for $30 \mathrm{~min}$. cDNA quality was verified by PCR amplification of $\beta$-actin.

RKIP mutational analysis. The complete coding region of RKIP was amplified by RT-PCR from cDNA using specific primers (RKIP108 for: ATGCCGGTGGACCTCAGC, RKIP657rev: GCTGCTCGTACAGTTTGGGC) resulting in a 546-bp fragment. The PCR reaction was performed in $50 \mu \mathrm{l}$ reaction volume containing $5 \mu 1$ 10X PCR buffer, $1 \mu 1 \mathrm{cDNA}$, $0.5 \mu 1$ of each primer $(0.2 \mu \mathrm{M}), 0.5 \mu 1 \mathrm{dNTPs}(10 \mathrm{mM}), 0.2 \mu \mathrm{l}$ Taq polymerase $(5 \mathrm{U} / \mu \mathrm{l})$, and water. The amplification reactions were performed by 35 repetitive cycles of denaturing for $1 \mathrm{~min}$ at $94^{\circ} \mathrm{C}$, annealing for $1 \mathrm{~min}$ at $58^{\circ} \mathrm{C}$, extension for $1 \mathrm{~min}$ at $72^{\circ} \mathrm{C}$, and a final extension step at $72^{\circ} \mathrm{C}$ for $5 \mathrm{~min}$. The PCR products were resolved on $1 \%$ agarose gels. For sequencing, the products were purified by PEG precipitation to remove unincorporated primers and dNTPs. Both strands were sequenced for each PCR product from at least two independent PCR reactions. Sequences were compared with 

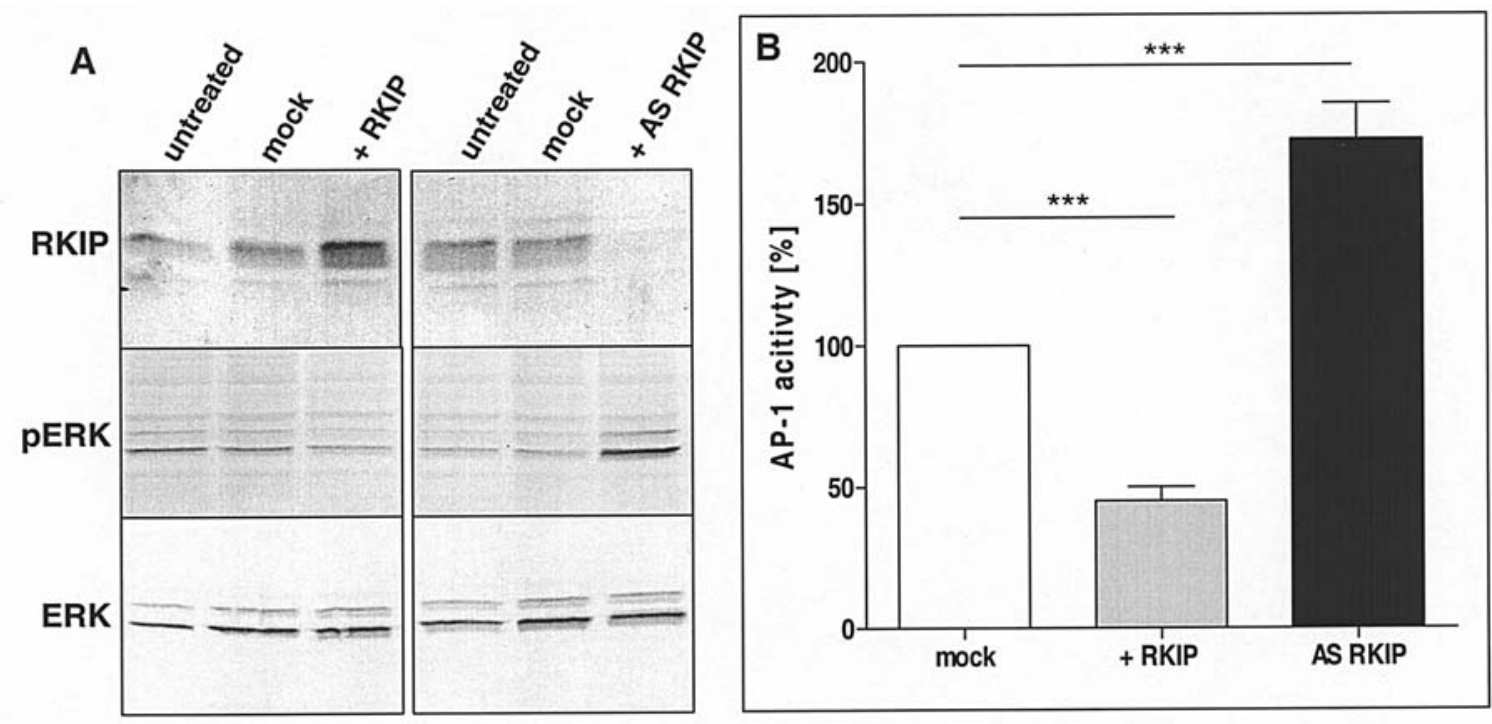

Figure 2. Effect of RKIP overexpression and RKIP reduction on ERK1/2 and AP-1 activity in HepG2 cells. (A) pERK1/2 levels in relation to ERK1/2 protein levels revealed reduced activity of ERK1/2 in RKIP overexpressing HepG2 cells (left panel), and higher activity of ERK1/2 in RKIP antisense transfected cells (right panel). The blots shown are representative examples. The experiments were repeated 3 times. (B) Luciferase reporter gene assays showed activation of AP-1 as target of Ras/Raf/MEK/ERK signalling to be significantly downregulated in RKIP overexpressing cells in comparison to mock transfected HepG2. In contrast, transfection of antisense RKIP construct led to an increase of AP-1 activity; ${ }^{* * *} \mathrm{P}<0.001$.

the gene data bank by means of BLAST search (National Center of Biotechnology Information, http://www.ncbi.nlm. nih.gov).

Analysis of RKIP expression by quantitative PCR. Quantitative real-time PCR was performed on a Lightcycler (Roche, Mannheim, Germany). cDNA (2 $\mu \mathrm{l}), 1.6 \mu 125 \mathrm{mM} \mathrm{MgCl}_{2}$, $0.2 \mu \mathrm{M}$ each of forward and reverse primers (RKIPfor455: CCTCCACCGCTATGTCTGGC, RKIP657rev: GCTGCC GTACAGTTTGGGC), $2 \mu 1$ SybrGreen Lightcycler mix in a total volume of $20 \mu \mathrm{l}$ were applied to the following PCR programme: $30 \mathrm{sec} 95^{\circ} \mathrm{C}$ (initial denaturation); $20^{\circ} \mathrm{C} / \mathrm{sec}$ temperature transition rate up to $95^{\circ} \mathrm{C}$ for $15 \mathrm{sec}, 3 \mathrm{sec} 64^{\circ} \mathrm{C}$, $5 \sec 72^{\circ} \mathrm{C}, 85^{\circ} \mathrm{C}$ acquisition mode single, repeated for 40 times (amplification). The PCR product was evaluated by melting curve analysis following the manufacturer's instructions and checking PCR products on $1.8 \%$ agarose gels. All quantitative PCR experiments were repeated at least 3 times.

Protein analysis in vitro (Western blotting). For protein isolation $2 \times 10^{6}$ cells were washed in $1 \mathrm{X}$ PBS and lysed in $200 \mu 1$ RIPA buffer (Roche). Protein concentration was determined using BCA protein assay reagent (Pierce, Rockford, USA). Equal amounts of total cellular protein were denatured at $70^{\circ} \mathrm{C}$ for $10 \mathrm{~min}$ after addition of Rotiload buffer (Roth, Karlsruhe, Germany) and separated on NuPage-SDS-gels (Invitrogen). Total protein $(20 \mu \mathrm{g})$ per lane were used for Western blot analyses. After transferring proteins onto PVDFmembranes (BioRad, Richmond, USA), membranes were blocked in 3\% BSA/PBS for $1 \mathrm{~h}$ and incubated with a 1:1500 dilution of primary polyclonal rabbit anti-RKIP antibody (9) overnight at $4^{\circ} \mathrm{C}$. For detection of pERK a monoclonal antiphospho p42/44 antibody (Cell Signaling, Beverly, USA) in a 1:2000 dilution was used. ERK levels were detected with anti-p42/44 polyclonal rabbit antibody (1:1000). Dilutions $(1: 1500)$ of anti-rabbit or anti-mouse IgG AP (Sigma) were used as secondary antibodies. Staining was performed using 5-bromo-4-chloro-3-indolyl phosphate/nitroblue tetrazolium tablets (Sigma). All Western blot experiments were repeated at least 3 times.

Immunohistochemistry. Paraffin-embedded tissue sections were screened for RKIP protein expression by immunohistochemistry. Tissues were deparaffinated, rehydrated, and incubated with primary polyclonal rabbit RKIP antibody $(1: 1500)$ overnight at $4^{\circ} \mathrm{C}$. The secondary antibody (biotinlabelled anti-rabbit, 1:1000, Jackson Immuno Research, West Grove, USA) was incubated for $30 \mathrm{~min}$ at room temperature, followed by incubation with streptavidin-peroxidase conjugate (Dako, Hamburg, Germany) for $30 \mathrm{~min}$. Antibody binding was visualised using 3-amino-9-ethylcarbazol-solution (Dako). Tissues were counterstained with haemalaun.

Statistical analyses. Data are presented as mean \pm standard deviation. Student's paired test was used to test differences between groups; P-values $<0.05$ were considered to be statistically significant. All statistical analyses were performed using the Prism 2.01 software (GraphPad Inc., San Diego, USA).

\section{Results}

Expression of RKIP in HCC cell lines and mutation analysis of RKIP $m R N A$. Expression levels of RKIP protein were detected by performing Western blot experiments, analysing three established HCC cell lines (PLC, HepG2, and Hep3B) and primary human hepatocytes (PHH), respectively. Fig. 1A shows a representative Western blot result. Reduced RKIP protein expression levels were observed in the $3 \mathrm{HCC}$ cell lines in comparison to PHH. In PLC and Hep3B cells downregulation was more pronounced than in HepG2 cells. Staining for $\beta$-actin was performed as control for equal protein loading. 


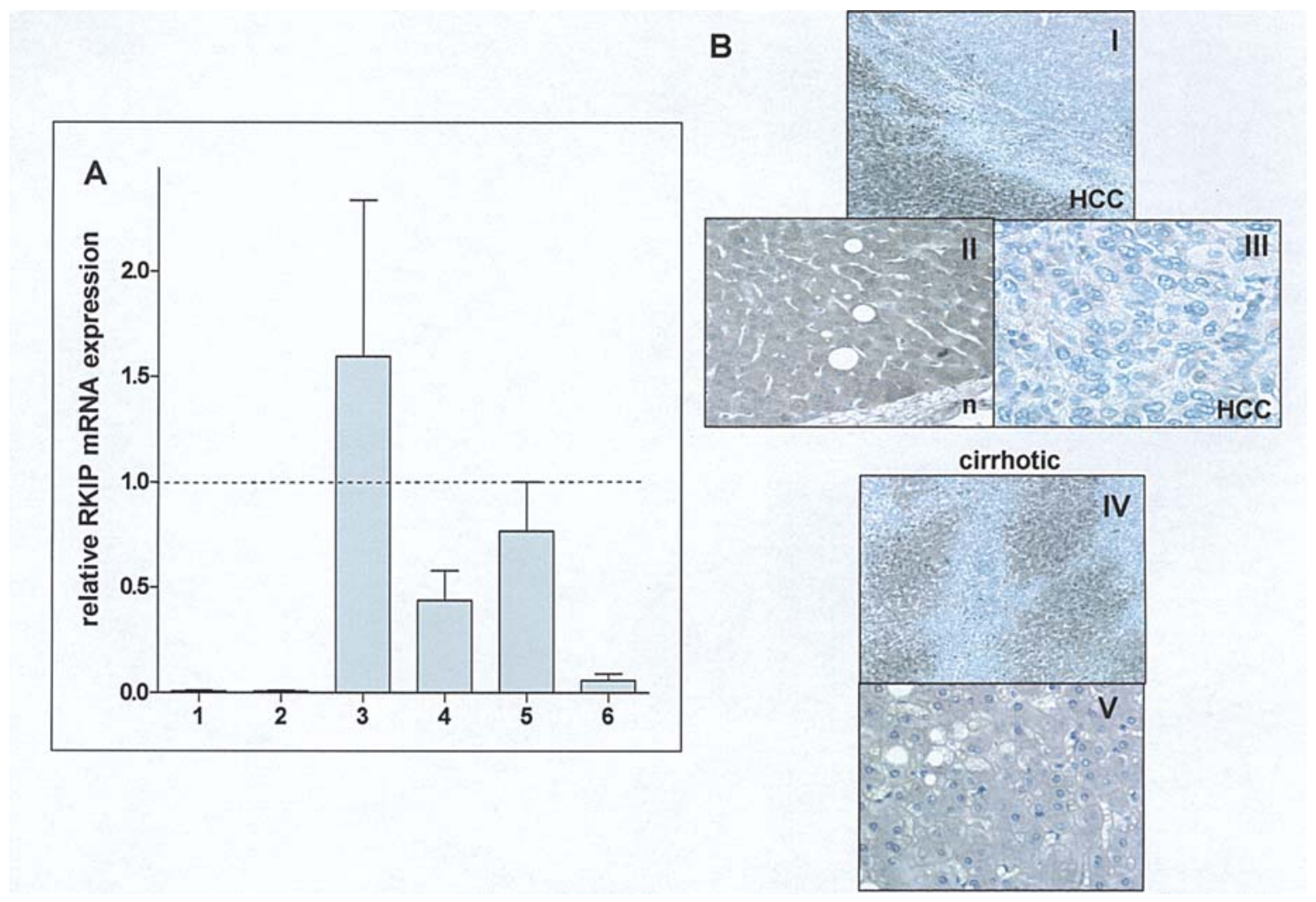

Figure 3. RKIP expression in hepatocellular carcinoma (HCC). (A) RKIP mRNA expression levels in HCC and corresponding non-tumourous tissue samples were analysed by quantitative RT-PCR showing RKIP mRNA expression levels in matched pairs of tumour and normal hepatic tissue. RKIP expression levels in HCC are shown in relation to adjacent non-tumourous tissue (set 1). (B) Tissue sections were immunostained with anti-RKIP antibody and developed in red (AEC). Slides were counterstained with haemalaun. HCC tumour and adjacent normal liver tissue are shown in panel I in x 50 magnification, representative stainings of non-tumourigenic (n) liver area and HCC area in x200 magnification are shown in panels II and III, respectively. Cirrhotic tissue (panels IV and V) is shown in $\times 50$ and $\times 200$ magnification, respectively.

To assess whether RKIP downregulation was already detectable on a transcriptional level, we performed quantitative RT-PCR analysis and results were in good agreement with protein levels (PLC 18\% $\pm 4.4, \mathrm{HepG} 239 \% \pm 2.8$, and Hep3B $13 \% \pm 1.0$ of RKIP mRNA levels in comparison to PHH, set 100\%) (Fig. 1B, white bars).

To examine whether mutations within the RKIP coding region had occurred in HCC cell lines, the complete RKIP coding region was amplified by RT-PCR. All HCC cell lines examined expressed RKIP mRNA at the expected length, indicating that no major deletions or mutational insertions had occurred. As control RKIP coding sequence of PHH was amplified (data not shown). All PCR products were purified and subsequently sequenced using two different primers. Analysis revealed no sequence variation within the coding region of the RKIP gene locus (data not shown).

As reduction of RKIP mRNA expression in HCC cell lines could not be explained by genomic losses or mutations of the RKIP gene, we hypothesised that promoter hypermethylation could have silenced gene expression. To test this hypothesis HCC cell lines were exposed to the demethylating agent 5azacytidine, and, subsequently, RKIP mRNA expression was quantified by real-time RT-PCR. 5-Azacytidine treatment did not induce RKIP expression (Fig. 1C, black bars), indicating that promoter hypermethylation does not account for RKIP downregulation in HCC cell lines.
Functional relevance of decreased RKIP expression levels. To analyse the function of RKIP in HCC cells, we overexpressed RKIP in the HCC cell line HepG2 by transfecting cells with an RKIP expression construct. In a second approach RKIP levels were further decreased by introducing an antisense RKIP construct. Successful overexpression or downregulation of RKIP, respectively, was confirmed by quantitative RT-PCR (data not shown) and Western blotting. In contrast, no changes of RKIP expression were seen in mock transfected controls (Fig. 2A).

Examination of ERK1/2 and phospho-ERK $1 / 2$ levels by Western blotting in control and RKIP transfected cells revealed downregulation of ERK activity in RKIP overexpressing cells in comparison to control cells (Fig. 2A left panel). In contrast, depletion of RKIP expression by transfection of antisense RKIP construct yielded an increase in ERK1/2 activity (Fig. 2A, right panel). Additionally, we tested activation of an AP-1 reporter construct in control, RKIP transfected, and antisense RKIP transfected HepG2 cells using luciferase reporter gene assays. We observed significantly reduced activity of the AP-1 reporter in cells transfected with RKIP expression plasmid in comparison to mock transfected cells, while a significant increase in AP-1 activity was found in cells with reduced RKIP expression (Fig. 2B: mock: 100\%, + RKIP: 45\% (5, AS RKIP: $172 \% \pm 12$, P-values $<0.001$ ). 
Analysis of RKIP protein expression in situ. We next performed experiments with HCC tissue samples to assess the question whether RKIP expression is altered also in HCC tumours in vivo and might therefore contribute to or support development and/or progression of HCC.

RKIP mRNA expression was analysed in HCC tissue and surrounding non-tumourous liver tissue by quantitative RTPCR experiments. Fig. 3A shows the results of 6 examined matched samples, displaying no profound alterations between tumour and adjacent non-cancerous tissue in two cases [numbers 3 and 5, relative RKIP levels were $1.59 \pm 0.54$ (patient 3), and 0.76 \pm 0.33 (patient 5), respectively]. However, in 4 patients moderate (number 4 ) or even strong (numbers 1 , 2 , and 6) downregulation of RKIP mRNA levels was found in HCC tumour samples.

We examined RKIP protein expression in vivo in $15 \mathrm{HCC}$ specimens by immunohistochemistry. In addition, two normal human liver samples were analysed. In these normal control liver hepatocytes showed strong cytoplasmatic staining (Fig. 3BI and II). Three tissue samples from patients diagnosed with HCC only displayed moderate reduction in RKIP expression between healthy and affected parts of the sample, while 12 HCC samples showed obvious and strong downregulation of RKIP expression in tumourigenic areas. Representative images are presented in Fig. 3BI and III.

RKIP immunohistochemistry was performed on cirrhotic liver tissues. Here, a heterogeneous staining pattern appeared. While some hepatocytes had lost RKIP expression, the majority of cells expressed RKIP. However, subcellular localisation of the protein is altered in cirrhotic areas. In contrast to normal tissue RKIP staining is not cytoplasmic, but membranous (Fig. 3BIV and V).

\section{Discussion}

The Ras/Raf/MEK/ERK signalling cascade is known to be an important pathway that regulates various cellular processes in response to extracellular signals. It has been shown before that mutations within proteins of this cascade can lead to malignant transformation of cells (19-21).

In the present study, we investigated the role of Raf kinase inhibitor protein (RKIP) in HCC. We and others have shown that RKIP downregulation is likely to contribute to development and progression of tumourigenic diseases such as malignant melanoma or prostate cancer $(16,17)$. However, no studies on RKIP regulation in HCC or other hepatic diseases have been reported.

Our investigations revealed no loss or mutation within the RKIP genomic region in HCC cell lines as RT-PCR spanning the entire RKIP coding sequence revealed products at the expected length and sequencing of the PCR products excluded mutations within the fragments. Moreover, no studies on mutations within the genomic region of RKIP on human chromosome 12q23 and its adjacent areas have been published to date, contributing to our hypothesis that chromosomal changes are not likely to lead to deregulation of RKIP expression in tumourigenic diseases. However, quantification of mRNA expression revealed a marked downregulation of RKIP expression in HCC cell lines in comparison to PHH. It is increasingly recognized that epigenetic mechanisms account for altered gene expression in HCC and, recently, we were able to show that downregulation of methylthioadenosin phosphorylase (MTAP) in HCC is due to hypermethylation of the promoter region (22). Therefore, we speculated that this epigenetic mechanism might also lead to RKIP downregulation in $\mathrm{HCC}$ as the RKIP promoter region harbours numerous CpG dinucleotides. However, RKIP downregulation in HCC appears not likely to be due to promoter hypermethylation as treatment of HCC cells with 5-azacytidine did not affect RKIP expression. Therefore, we hypothesize that altered transcriptional regulation is responsible for reduced RKIP expression in HCC.

Functional analyses revealed that overexpression of RKIP in HepG2 cells leads to decreased ERK1/2 and AP-1 activity, while the opposite effect was observed in cells with reduced RKIP expression. These results are in good agreement with the initially described function of RKIP as inhibitor of the ERK signalling pathway $(13,14)$. However, several attempts to generate stably RKIP expressing cells failed in three different HCC cell lines (data not shown). Based on this observation we speculate that stable re- or overexpression of RKIP in these cells leads to modified cellular features causing elimination of highly RKIP expressing cells, at least in our experimental conditions.

In vitro analyses were supported and confirmed by in situ experiments on mRNA and protein level. Immunostaining of HCC tumours revealed downregulation of RKIP expression in most cases. As enhanced ERK1/2 activity can be observed in a number of hepatocellular carcinomas and has been reported to be correlated with tumour progression $(9,10,12)$, reduced expression of the endogenous inhibitor RKIP in those tumours could contribute to or even account for this effect.

As mentioned earlier, mutations in Ras, Raf, MEK, and ERK were identified in a variety of tumour entities and it has been shown that ERK is activated in a high number of hepatocellular tumours (9-12). Tannapfel and coworkers have studied BRAF mutations in HCC but did not detect BRAF mutations (23), supporting our hypothesis that deregulation of RKIP rather than genetic mutations might account for increased ERK activity in HCC.

In recent studies, we and others $(16,17)$ have shown that decreased RKIP expression is associated with tumourigenic transformation of cells. Therefore, in addition to HCC, we analysed RKIP expression in cirrhotic human liver. Cirrhotic liver tissues revealed altered subcellular localisation of RKIP. Membranes of hepatocytes were intensely stained, but the cytoplasm was almost negative for RKIP. It has been described by Liu and coworkers that in cirrhosis Ras gets activated (24) and Harding et al have described that during cirrhotic conversion of liver tissue the Ras/Raf pathway is activated and Raf gets recruited to the cell membrane (25). We speculate that due to interaction with membrane associated Raf in cirrhosis, RKIP localises to the inner surface of the cell membranes of hepatocytes, whereas in carcinogenic transformed cells, RKIP expression is downregulated.

In summary, we conclude that downregulation of RKIP observed in hepatocellular carcinoma may contribute to elevated ERK activity. Herewith, loss of RKIP may serve as a risk marker for HCC development and progression and 
re-expression of RKIP may be a potential, innovative therapeutic strategy for HCC.

\section{Acknowledgements}

We thank Walter Kolch, Glasgow, UK, for generously providing anti-RKIP antibody. This work was supported by grants from the DFG and the Deutsche Krebshilfe to A.B.

\section{References}

1. Okuda K: Hepatocellular carcinoma. J Hepatol 32: 225-237, 2000.

2. Schafer DF and Sorrell MF: Hepatocellular carcinoma. Lancet 353: 1253-1257, 1999 .

3. Thorgeirsson SS and Grisham JW: Molecular pathogenesis of human hepatocellular carcinoma. Nat Genet 31: 339-346, 2002.

4. Coleman WB: Mechanisms of human hepatocarcinogenesis. Curr Mol Med 3: 573-588, 2003.

5. Nottage M and Siu LL: Rationale for Ras and raf-kinase as a target for cancer therapeutics. Curr Pharm Des 8: 2231-2242, 2002.

6. O'Neill E and Kolch W: Conferring specificity on the ubiquitous Raf/MEK signalling pathway. Br J Cancer 90: 283-288, 2004.

7. Chang F, Steelman LS, Lee JT, et al: Signal transduction mediated by the Ras/Raf/MEK/ERK pathway from cytokine receptors to transcription factors: potential targeting for therapeutic intervention. Leukemia 17: 1263-1293, 2003.

8. Smalley KS: A pivotal role for ERK in the oncogenic behaviour of malignant melanoma? Int J Cancer 104: 527-532, 2003.

9. Hsiang CY, Wu SL and Ho TY: Activation of activator protein 1 and extracellular signal-regulated kinases in human hepatocellular transformation. Tumour Biol 25: 313-320, 2004.

10. Ito Y, Sasaki Y, Horimoto M, et al: Activation of mitogenactivated protein kinases/extracellular signal-regulated kinases in human hepatocellular carcinoma. Hepatology 27: 951-958, 1998.

11. Schmidt CM, McKillop IH, Cahill PA and Sitzmann JV: Increased MAPK expression and activity in primary human hepatocellular carcinoma. Biochem Biophys Res Commun 236: 54-58, 1997.

12. Tsuboi Y, Ichida T, Sugitani S, et al: Overexpression of extracellular signal-regulated protein kinase and its correlation with proliferation in human hepatocellular carcinoma. Liver Int 24: 432-436, 2004.
13. Yeung K, Seitz T, Li S, et al: Suppression of Raf-1 kinase activity and MAP kinase signalling by RKIP. Nature 401: 173-177, 1999.

14. Yeung K, Janosch P, McFerran B, et al: Mechanism of suppression of the Raf/MEK/extracellular signal-regulated kinase pathway by the raf kinase inhibitor protein. Mol Cell Biol 20: 3079-3085, 2000.

15. Serre L, Pereira DJ, Zelwer C, Bureaud N, Schoentgen F and Benedetti H: Crystal structures of YBHB and YBCL from Escherichia coli, two bacterial homologues to a Raf kinase inhibitor protein. J Mol Biol 310: 617-634, 2001.

16. Fu Z, Smith PC, Zhang L, et al: Effects of raf kinase inhibitor protein expression on suppression of prostate cancer metastasis. J Natl Cancer Inst 95: 878-889, 2003.

17. Schuierer MM, Bataille F, Hagan S, Kolch W and Bosserhoff AK: Reduction in Raf kinase inhibitor protein expression is associated with increased Ras-extracellular signal-regulated kinase signaling in melanoma cell lines. Cancer Res 64: 5186-5192, 2004.

18. Weiss TS, Jahn B, Cetto M, Jauch KW and Thasler WE: Collagen sandwich culture affects intracellular polyamine levels of human hepatocytes. Cell Prolif 35: 257-267, 2002.

19. Davies H, Bignell GR, Cox C, et al: Mutations of the BRAF gene in human cancer. Nature 417: 949-954, 2002.

20. Ward Y, Wang W, Woodhouse E, Linnoila I, Liotta L and Kelly K: Signal pathways which promote invasion and metastasis: critical and distinct contributions of extracellular signal-regulated kinase and Ral-specific guanine exchange factor pathways. Mol Cell Biol 21: 5958-5969, 2001.

21. Welch DR, Sakamaki T, Pioquinto R, et al: Transfection of constitutively active mitogen-activated protein/extracellular signal-regulated kinase kinase confers tumorigenic and metastatic potentials to NIH3T3 cells. Cancer Res 60: 1552-1556, 2000.

22. Hellerbrand C, Muhlbauer M, Wallner S, et al: Promotorhypermethylation is causing functional relevant downregulation of methylthioadenosine phosphorylase (MTAP) expression in hepatocellular carcinoma. Carcinogenesis 27: 64-72, 2006.

23. Tannapfel A, Sommerer F, Benicke M, et al: Mutations of the BRAF gene in cholangiocarcinoma but not in hepatocellular carcinoma. Gut 52: 706-712, 2003.

24. Liu P, Flejou JF, Feldmann G and Bernuau D: Activation of ras oncogene in livers with cirrhosis. J Hepatol 21: 1103-1108, 1994.

25. Harding A, Tian T, Westbury E, Frische E and Hancock JF: Subcellular localization determines MAP kinase signal output. Curr Biol 15: 869-873, 2005. 